\title{
Follow-up of cancer in primary care versus secondary care: systematic review
}

Ruth A Lewis, Richard D Neal, Nefyn H Williams, Barbara France, Maggie Hendry, Daphne Russell, Dyfrig A Hughes, Ian Russell, Nicholas SA Stuart, David Weller and Clare Wilkinson

\begin{abstract}
Background

Cancer follow-up has traditionally been undertaken in secondary care, but there are increasing calls to deliver it in primary care.

Aim

To compare the effectiveness and cost-effectiveness of primary versus secondary care follow-up of cancer patients, determine the effectiveness of the integration of primary care in routine hospital follow-up, and evaluate the impact of patient-initiated follow-up on primary care.

Design of study

Systematic review.

Setting

Primary and secondary care settings.

Method

A search was carried out of 19 electronic databases, online trial registries, conference proceedings, and bibliographies of included studies. The review included comparative studies or economic evaluations of

Following completion of treatment, most cancer patients are followed up regularly in hospital outpatient clinics. The perceived benefit of this is to facilitate diagnosis of recurrent disease, monitor the effectiveness and side-effects of treatment, manage comorbidity, and identify and treat psychosocial problems. $^{1-4}$ There is also evidence that patients value the psychological and social support that cancer follow-up provides, ${ }^{5-8}$ and find it reassuring. ${ }^{9-11}$ Conversely, hospital follow-up might also prompt unnecessary tests, raise anxiety, provide false reassurance, and delay the patient's return to full function. For some cancer sites, such as breast and colorectal cancer, there is good evidence that routine follow-up does not provide survival benefit or lead to earlier diagnosis of recurrences, other than in terms of detecting locoregional recurrence or contralateral new primaries. ${ }^{12-16}$
\end{abstract} primary versus secondary care follow-up, hospital follow-up with formal primary care involvement versus conventional hospital follow-up, and hospital follow-up versus patient-initiated or minimal follow-up if the study reported the impact on primary care.

Results

There was no statistically significant difference for patient wellbeing, recurrence rate, survival, recurrencerelated serious clinical events, diagnostic delay, or patient satisfaction. GP-led breast cancer follow-up was cheaper than hospital follow-up. Intensified primary health care resulted in increased home-care nurse contact, and improved discharge summary led to increased GP contact. Evaluation of patient-initiated or minimal follow-up found no statistically significant impact on the number of GP consultations or cancerrelated referrals.

\section{Conclusion}

Weak evidence suggests that breast cancer follow-up in primary care is effective. Interventions improving communication between primary and secondary care could lead to greater GP involvement. Discontinuation of formal follow-up may not increase GP workload. However, the quality of the data in general was poor, and no firm conclusions can be reached.

Keywords

long-term care; neoplasms; outpatients; primary health care; systematic review.

\section{INTRODUCTION}

RA Lewis, MSc, BSc, lecturer; RD Neal, PhD, FRCGP, senior clinical lecturer; $N H$ Williams, $P h D$, FRCGP, senior clinical lecturer; B France, research assistant; $\boldsymbol{M}$ Hendry, BA Research fellow; C Wilkinson, MD, FRCGP, Department of Primary Care and Public Health, North Wales Clinical School, School of Medicine, Cardiff University, Wrexham. D Russell, PhD, senior research fellow/trial statistician, Institute for Medical and Social Care Research; NSA Stuart, DM, FRCP, North Wales Clinical School; DA Hughes, PhD reader, Centre for Economics and Policy in Health, Bangor University, Bangor, Bangor. I Russell, PhD, FRCGP, FRCP(Ed), professor, School of Medicine, Swansea University, Swansea. D Weller, PhD, MRCGP, FRCP, professor, Department of General Practice, University of Edinburgh, Edinburgh.

\section{Address for correspondence}

Ruth Lewis, Cardiff University, Department of Primary Care and Public Health, School of Medicine, North Wales Clinical School, Gwenfro Building Unit 6/7, Wrexham Technology Park, Wrexham, LL13 7YP. E-mail: lewisr17@cf.ac.uk

Submitted: 3 November 2008; Editor's response: 6 January 2009; final acceptance: 6 February 2009.

(C) British Journal of General Practice

This is the full-length article of an abridged version published in print. Cite this article as: Br J Gen Pract 2009; DOI: 10.3399/bjgp09X453567. 
Follow-up of cancer accounts for a substantial burden of outpatient activity. Financial and other drivers are putting downward pressure on 'routine' secondary care follow-up, and new models of care are developing, often through cancer collaboratives. ${ }^{17}$ In the UK, primary care, with its universal system of patient registration, generalist skills, and high satisfaction ratings, may be well placed to undertake some of this work. The UK general medical services contract encourages the review of cancer patients by including this within the Quality and Outcomes Framework. Some low-risk follow-up is already done in primary care, notably in prostate cancer, and more is advocated. ${ }^{18-20}$ However, this seems to be happening without rigorous evaluation. There is some disagreement between specialists and GPs about where care should be delivered, and debate about patient preference. ${ }^{21}$

A systematic review was conducted to compare the effectiveness and cost-effectiveness of primary versus secondary care follow-up of cancer patients. The study also evaluated the impact on primary care of discontinuing formal follow-up or replacing it with patient-initiated follow-up, as well as interventions integrating primary and secondary care for cancer follow-up. Qualitative studies were also included, but their findings are reported separately. ${ }^{22}$ (The review also looked at nurse-led follow-up as indicated in Figure 1, the findings of which are reported elsewhere. ${ }^{23}$ )

\section{METHOD}

The following databases were searched (from inception to February 2007) using strategies designed specifically for each database: MEDLINE, MEDLINE in process, EMBASE, CINAHL, PsychINFO, AMED, BIOSIS, Index to Scientific and Technical proceedings, Science Citation Index, Social Science Citation Index, Cochrane Central Register of Controlled Trials, Database of Abstracts of Reviews of Effects, Cochrane Database of Systematic Reviews, Health Technology Assessment database, NHS Economic Evaluation database, System for Information on Grey Literature, British Nursing Index, Health Management Information Consortium, National Research Register, and other trial registries $(n=7)$ available via the internet. No language restriction was used.

Each cancer site was searched separately, and full details of the search strategy are available on request and described elsewhere (R Lewis et al, unpublished data, 2007). (An example of the search strategy used for breast cancer is presented in Appendix 1.) Additional references were identified through reviewing the bibliographies of 16 retrieved systematic reviews and 42 included studies, and hand searching five conference proceedings.

\section{How this fits in}

Amid increasing debate, cancer follow-up is being shifted to primary care settings without rigorous evaluation. GP follow-up for breast cancer may be effective. A more formal involvement of primary care in routine hospital follow-up could lead to improved communication between primary and secondary care and more patients using GPs for support. Discontinuation of formal routine hospital follow-up does not appear to have an impact on primary care workload.

The search included any type of study or economic evaluation that compared cancer followup in primary care with that in a secondary setting. It also included studies that examined any type of intervention that involved formal primary care input in

Figure 1. Flow diagram showing the number of references identified, retrieved, and included in the review.

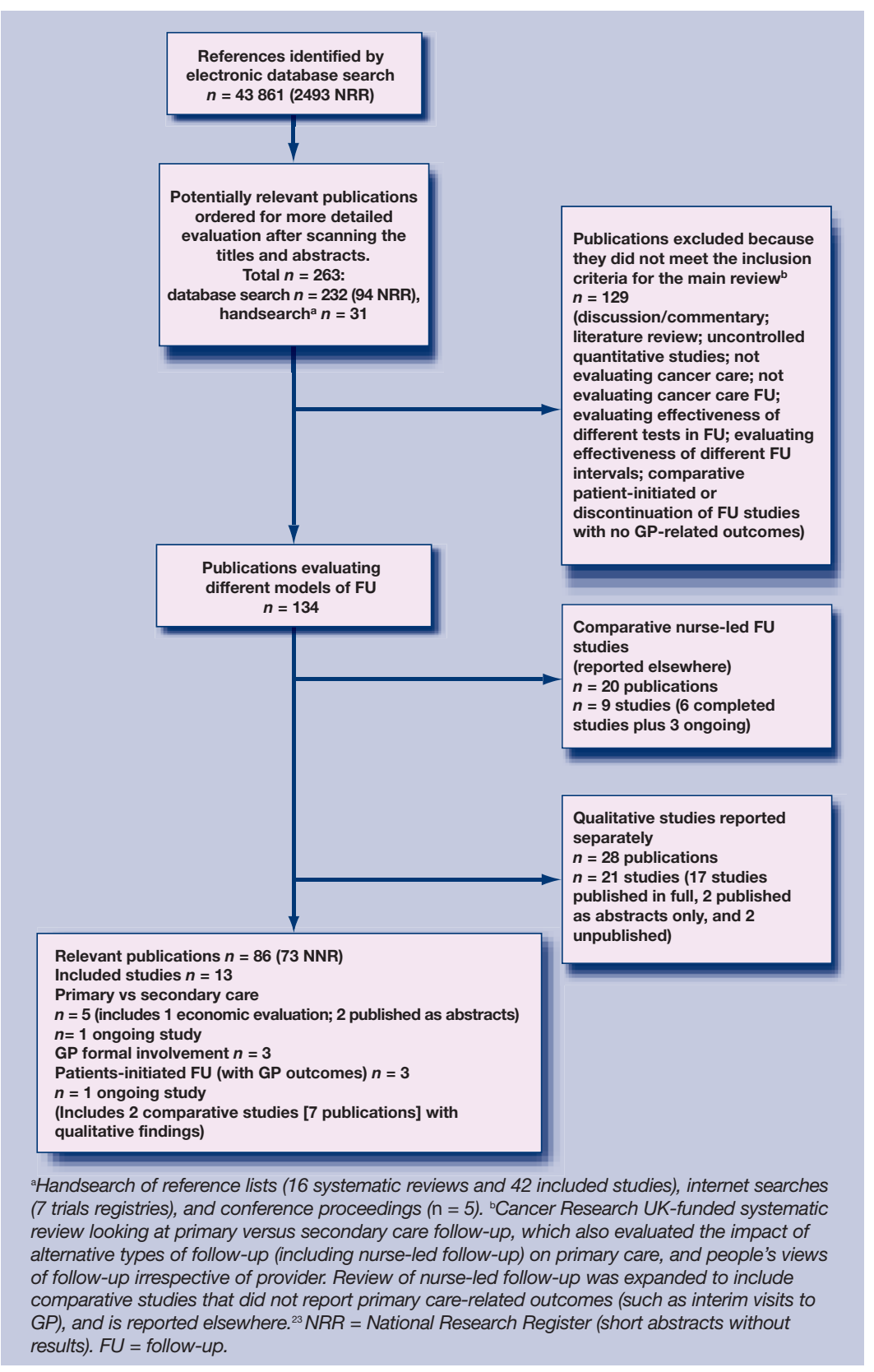


routine hospital follow-up. Any type of outcome measure was included. Studies that compared follow-up with no follow-up or patient-initiated follow-up were also considered, but only if they reported data on primary care-related outcomes. The population of interest included patients of any age who had received treatment for any type and stage of cancer. Only studies that examined the follow-up of patients who were free of active disease or no longer receiving treatment for the following purposes were included: to identify recurrent tumours of new primary disease; to provide support for complications or delayed side-effects of treatments; or to identify patients who may require additional help or treatments (for example, for functional or psychological problems). Studies that examined patients who were still receiving hospital-based treatment (for example, radiotherapy) or treatment after care, rehabilitation, or specialist palliative care were excluded. Patients in cancer follow-up but receiving long-term therapy, such as hormonal treatment for breast or prostate cancer, who did not require frequent or routine hospital visits and who were free of active disease were included.

Two reviewers independently assessed the results of each cancer site-specific literature search and the relevance of retrieved studies. Data were extracted by one reviewer, using a predefined form, and checked by a second independent reviewer. Quality assessments were conducted independently by two reviewers. Disagreements were resolved by discussion and, when necessary, a third reviewer was consulted.

The quality of effectiveness studies was assessed using the checklist developed by Downs and Black, ${ }^{24}$ modified according to the suggestions made by Deeks et al,,$^{25}$ and adapted for use on cancer followup studies. Economic evaluations were assessed using an updated version of the checklist developed by Drummond and Jefferson. ${ }^{26}$

Due to variation in the way the outcome data were reported and analysed, a meta-analysis was not feasible, even for the same outcome measures within each follow-up group. A narrative synthesis was therefore conducted.

\section{RESULTS}

The electronic searches identified 43861 references of which 232 papers were retrieved in full. Thirty-one additional studies were identified by hand searching (Figure 1).

\section{Primary versus secondary care}

Five studies compared primary versus secondary care follow-up,,$^{3,27-30}$ but two were only reported in abstract form (a non-randomised study of patients who had undergone haematopoietic stem cell transplantation for malignant haematologic disorders ${ }^{28}$ and a randomised controlled trial [RCT] of patients with cutaneous melanoma $\left.{ }^{29}\right)$. The three published RCTs examined follow-up for breast cancer $^{3,27}$ and colon cancer ${ }^{30}$ (Table 1, part a). One of the breast cancer studies was a non-inferiority trial, ${ }^{27}$ and the other incorporated a cost analysis. ${ }^{3}$ Two studies recruited patients who had recently completed primary treatment, ${ }^{27,30}$ and one study recruited women who were already receiving followup. $^{3}$ The percentages of eligible participants who declined to be randomised to primary or secondary care follow-up were fairly high $\left(33 \%,{ }^{3} 40 \%,{ }^{30}\right.$ and $55 \%{ }^{27}$ ), especially for studies that recruited patients who had just completed treatment. ${ }^{27,30}$ Only one study (breast cancer) incorporated a pre-trial education session of cancer follow-up for GPs, who were also given a handbook and discharge summary information. ${ }^{3}$ In the remaining two studies, GPs were provided with a brief summary of the current followup guidance.

The three RCTs that examine primary care versus secondary care follow-up ${ }^{3,2,30}$ were well conducted (Table 2, part a). All used an adequate randomisation method with allocation concealed from patients and clinicians. An attempt was made to blind those measuring the main outcomes in all three studies. The sample size was fairly small in two studies (range $n=203^{30}$ to $296^{3}$ ), and large in the non-inferiority trial ( $n=968) .{ }^{30}$ The length of follow-up ranged from 18 months ${ }^{30}$ to a median of 3.5 years. ${ }^{27}$

There were no statistically significant differences between the intervention groups in terms of patient wellbeing (psychological morbidity and quality of life), recurrence rate, or survival, but this may be due to lack of statistical power and the short duration of the studies (Table 3 and Table 4). The non-inferiority trial found no statistically significant difference between the groups for the main outcome of recurrence-related serious clinical events (defined as spinal cord compression, pathological fracture, hypercalcaemia, uncontrolled local recurrence, brachial plexopathy, or poor functional status at the time of diagnosis of recurrence), and was unable to demonstrate statistically significant non-inferiority. ${ }^{27}$ Although the absolute difference between the intervention groups was small (1.9\%), the observed lower band of the confidence interval $(95 \% \mathrm{Cl}=$ -2.26 to 2.65 ) crossed the non-inferiority margin of $1.5 \%$. The patient population included women with early-stage breast cancer $(69 \%$ had stage I-II disease) for whom a serious clinical event is a rare outcome, and in whom the length of follow-up (median 3.5 or 4.5 years post diagnosis; $31 \%$ had five-year follow-up) may not have been sufficient to 


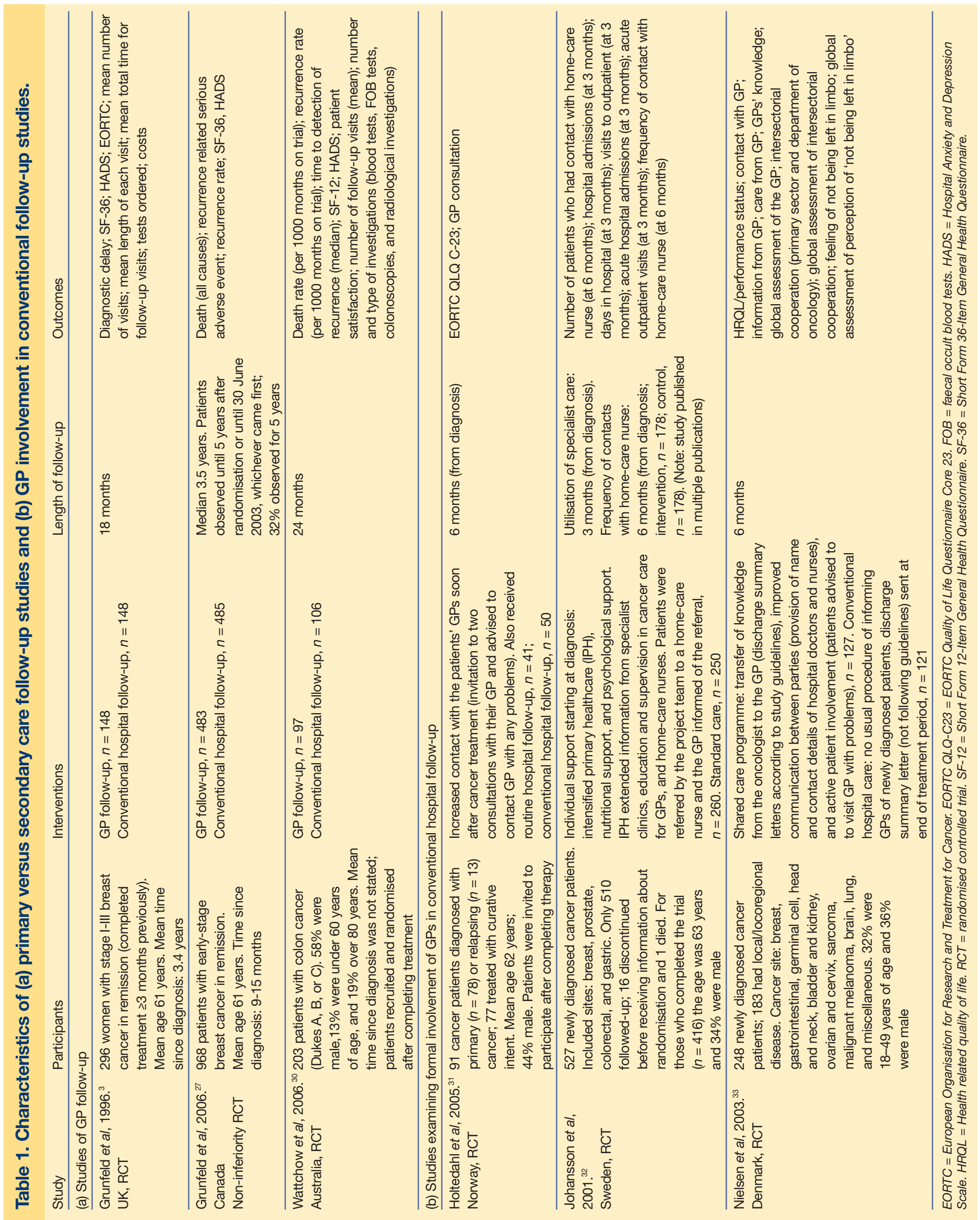


Table 2. Quality of included studies comparing primary- versus secondary-care follow-up.

\begin{tabular}{|c|c|c|c|c|c|c|}
\hline \multirow[b]{2}{*}{ Downes and Black ${ }^{24}$ item score (maximum score) } & \multicolumn{3}{|c|}{ Part a } & \multicolumn{3}{|c|}{ Part b } \\
\hline & $\begin{array}{l}\text { Grunfeld } \\
t \text { al, 2006, }{ }^{27} \\
\text { breast } \\
\text { cancer }\end{array}$ & $\begin{array}{c}\text { Grunfeld } \\
\text { et al, } 1996,{ }^{3} \\
\text { breast } \\
\text { cancer }\end{array}$ & $\begin{array}{l}\text { Wattchow } \\
\text { et al, 2006, } \\
\text { colon } \\
\text { cancer }\end{array}$ & $\begin{array}{l}\text { Holtedahl } \\
\text { et al, } 2005,{ }^{31} \\
\text { multiple } \\
\text { site cancer }\end{array}$ & $\begin{array}{l}\text { Johansson } \\
\text { et al, 2001, }{ }^{32} \text { et } \\
\text { multiple site } \\
\text { cancer s s }\end{array}$ & $\begin{array}{c}\text { Nielsen } \\
\text { et al, 2003, } \\
\text { multiple } \\
\text { site cancer }\end{array}$ \\
\hline \multicolumn{7}{|l|}{ Reporting } \\
\hline Is the aim of the study clearly described? (1) & 1 & 1 & 1 & 1 & 1 & 1 \\
\hline Are the outcomes to be measured clearly described? (1) & 1 & 1 & 1 & 1 & 1 & 1 \\
\hline Are the characteristics of participants clearly described? (1) & 1 & 1 & 1 & 1 & 1 & 1 \\
\hline Are the interventions clearly described? (1) & 1 & 1 & 1 & 1 & 1 & 1 \\
\hline Are the distributions of principal confounders in each group described? (2) & 2 & 2 & 1 & 1 & 1 & 2 \\
\hline Are the main findings clearly described? (1) & 0 & 1 & 1 & 0 & 1 & 1 \\
\hline Can the reader calculate estimates of variability in data for the main outcomes? (1) & 1 & 1 & 1 & 1 & 0 & 0 \\
\hline Have all important adverse effects been reported? (1) & 1 & 1 & 1 & 1 & 1 & 0 \\
\hline Have the characteristics of participants lost to follow-up been described? (1) & 1 & 1 & 1 & 0 & 1 & 0 \\
\hline Have confidence intervals or exact significance levels been reported? (1) & 1 & 1 & 1 & 0 & 0 & 1 \\
\hline \multicolumn{7}{|l|}{ External validity } \\
\hline Were those who were asked to participate, representative of their population? (1) & 1 & 1 & 1 & 0 & 0 & 1 \\
\hline Were those who agreed to participate representative of their population? (1) & 0 & 1 & 0 & 0 & 0 & 1 \\
\hline Were the staff/setting/facilities representative of those the majority receive? (1) & 1 & 1 & 1 & 1 & 1 & 1 \\
\hline \multicolumn{7}{|l|}{ Internal validity: bias } \\
\hline Was there an attempt to blind those measuring the main outcomes? (1) & 1 & 1 & 1 & 1 & 0 & 1 \\
\hline If any results were based on 'data dredging', was this made clear? (1) & 0 & 1 & 1 & 0 & 0 & 0 \\
\hline Do analyses adjust for different lengths of follow-up? (1) & 0 & 0 & 1 & 1 & 1 & 1 \\
\hline Was the length of follow-up adequate? (1) & 1 & 1 & 1 & 0 & 1 & 1 \\
\hline Were the statistical tests used appropriate? (1) & 1 & 1 & 1 & 1 & 1 & 0 \\
\hline Was non-compliance reported appropriately? (1) & 1 & 1 & 1 & 1 & 1 & 1 \\
\hline Were the main outcome measures used valid and reliable? (1) & 1 & 0 & 1 & 1 & 1 & 1 \\
\hline \multicolumn{7}{|l|}{ Internal validity: confounding (selection bias) } \\
\hline Were control and intervention participants recruited from the same population? (1) & (1) 1 & 1 & 1 & 1 & 1 & 1 \\
\hline Were participants recruited over the same time period? (1) & 1 & 1 & 1 & 1 & 1 & 1 \\
\hline Were study participants randomised to intervention groups? (2) & 2 & 2 & 2 & 2 & 2 & 2 \\
\hline Was randomisation concealed from participants and staff until after recruitment? (1) & (1) 1 & 1 & 1 & 0 & 0 & 0 \\
\hline Was there adjustment for confounding in the analyses? (2) & 1 & 0 & 1 & 0 & 1 & 0 \\
\hline Were losses to follow-up taken into account? (1) & 1 & 1 & 1 & 0 & 0 & 1 \\
\hline \multicolumn{7}{|l|}{ Power } \\
\hline Has an estimate of clinically important difference been specified? (2) & 1 & 1 & 1 & 0 & 1 & 0 \\
\hline Is the sample size adequate? (3) & 1 & 1 & 2 & 0 & 2 & 1 \\
\hline Total score (maximum 34) & 26 & 27 & 29 & 17 & 22 & 22 \\
\hline
\end{tabular}

Numbers in brackets indicate highest possible score.

measure the serious effects of recurrent disease. Although patients remained in the trial until death, assessment for serious clinical outcomes was only done until a recurrence was detected or the trial ended.

Only two studies examined patient satisfaction. ${ }^{3,30}$ One found that satisfaction with breast cancer follow-up was higher in the GP group (6 of 12 items) than the hospital group at 9 months (mid trial), ${ }^{3}$ but did not report satisfaction at 18-month follow-up (end of trial). The second study reported no statistically significant difference between the groups at 2 years for colon cancer follow-up. ${ }^{30}$ Two studies also evaluated resource use..$^{3,30}$ Hospital doctors and GPs were found to differ in the type and number of diagnostic tests ordered, ${ }^{3,30}$ as well as the length and frequency of visits. ${ }^{3}$ A comprehensive cost analysis (cost year 1994) found that breast cancer follow-up by the GP was less costly than routine hospital follow-up, by a mean of $£ 130$ per patient $(95 \% \mathrm{Cl}=$ $£ 112$ to £149). This was due to a difference in physician cost and not because of the variation in diagnostic tests ordered. ${ }^{3}$

\section{Formal involvement of GP in cancer care follow-up versus conventional care}

Three RCTs examined formal involvement of primary care in routine hospital follow-up. ${ }^{31-33}$ No economic evaluations were identified. All three studies included patients with cancer originating from multiple sites (Table 1, part b). The study by Holtedahl et al examined an intervention that involved patients being 
Table 3. Results of survival and recurrence.

\begin{tabular}{|c|c|c|}
\hline Study details & Survival & Recurrence \\
\hline $\begin{array}{l}\text { Grunfeld et al, } 1996 .^{3} \\
\text { Cancer site: breast. } \\
\text { Length of follow-up: } \\
18 \text { months. Sample size: } \\
n=296 \text {; GP } n=148, \\
\text { hospital } n=148\end{array}$ & Not reported & $\begin{array}{l}\text { Diagnostic delay: GP } n=22 \text { versus hospital } n=21 \text {; } \\
\text { mean difference } 1.5(95 \% \mathrm{Cl}=-13 \text { to } 22) \text {. Assessed at } \\
\text { a single date: recurrence rate GP } n=10(6.8 \%) \text { versus } \\
\text { hospital } n=16(10.8 \%) \text {. No comprehensive review at } \\
\text { end of trial to identify missed recurrences }\end{array}$ \\
\hline $\begin{array}{l}\text { Grunfeld et al, } 2006^{27} \\
\text { Cancer site: breast. Length } \\
\text { of follow-up: median } 3.5 \text { years. } \\
\text { Sample size: } n=968 \text {; } \\
\text { GP } n=483 \text {, hospital } n=485\end{array}$ & $\begin{array}{l}\text { Deaths (all cause): GP } 29(6.0 \%) \text {, versus hospital } 30 \\
(6.2 \%) \text {; difference: } 0.18 \%(95 \% \mathrm{Cl}=-2.90 \text { to } 3.26)\end{array}$ & $\begin{array}{l}\text { Recurrence-related serious clinical events: GP } \\
n=17(3.5 \%) \text { versus hospital } n=18(3.7 \%) \text {; difference: } \\
0.19 \%(95 \% \mathrm{Cl}=-2.26 \text { to } 2.65) \text {. Recurrence or new } \\
\text { contralateral breast cancers: GP } n=54(11.2 \%) \text { versus } \\
\text { hospital } n=64(13.2 \%) \text {; difference: } 2.02 \%(95 \% \mathrm{Cl}= \\
-2.13 \text { to } 6.16) \text {. Comprehensive review at end of trial } \\
\text { found no missed recurrence }\end{array}$ \\
\hline $\begin{array}{l}\text { Wattchow et al, } 2006^{30} \\
\text { Cancer site: colon. Length } \\
\text { of follow-up: } 24 \text { months. } \\
\text { Sample size: } n=203 \text {; } \\
\text { GP } n=97 \text {, hospital } n=106\end{array}$ & $\begin{array}{l}\text { Death rates (per } 1000 \text { months on trial): GP } n=6.6 \\
\text { versus hospital } n=5.4 ; P=0.67 \text {, Fisher's exact test. } \\
\text { Median survival (months): GP } 31 \text { versus hospital } 20 \text {; } \\
P=0.69 \text {, log rank test }\end{array}$ & $\begin{array}{l}\text { Recurrence rate (per } 1000 \text { months on trial): GP } n=7.1 \\
\text { versus hospital } 8.0 ; P=0.92 \text {, Fisher's exact test. } \\
\text { Median time to detection (months): GP } n=9.5 \text { versus } \\
\text { hospital } 8.0 ; P=0.76 \text {, log rank test. Recurrence rate } \\
\text { obtained from state-based cancer registries }\end{array}$ \\
\hline
\end{tabular}

invited to attend two 30-minute consultations with their GP: one soon after completing treatment and the other 6 months later. ${ }^{31}$ During the consultation, patients were asked about their wellbeing and their experience of having cancer (open-ended questions provided by researchers). Patients were also advised to contact their GP if they had any cancer-related queries or problems. In addition, patients received routine hospital follow-up; the control group received routine hospital follow-up only.

The study by Johansson et al examined an intervention that was part of the Support-CareRehabilitation project, which involved individual patient support in terms of intensified primary health care, nutritional support, and psychological support. ${ }^{32}$ The intervention was implemented as soon as possible after randomisation, and patients were referred to a home-care nurse, who contacted the patient and suggested follow-up contacts during the period of primary treatment and rehabilitation or palliative care. GPs of these patients were informed of the cancer diagnosis and the referral to the homecare nurse. Intensified primary health care involved extended information from the specialist clinics (GPS and home-care nurses received copies of the medical records each time the patient was discharged from hospital after a period of inpatient care or attended outpatient clinic); education (in diagnostics and treatments of cancer, pain and diet management, psychosocial support, and palliative care); and supervision in cancer care for GPs and home-care nurses. The control included standard care, which routinely did not include follow-up contacts made by home-care nurses.

Nielsen et al examined an intervention which involved the use of discharge letters that followed predefined guidelines, which were developed for the study. ${ }^{33}$ These included details of the investigations, treatment, and information the patient had received; described in detail which physical, psychological, and social problems the patient had or might expect to get; contained information about what the oncologist expected the GP to do; and provided information about the patient's type of cancer, treatment plans, and prognosis as well as information about treatment of common side effects and pain. The names and phone numbers of the hospital doctors and nurses who were responsible for the patient were also attached to the discharge letters. Patients received both oral and written information about the information package given to their GPs, and were advised to contact their GP if they had any problems they thought could be solved in this setting. The control group received routine hospital care, where the GP was rarely informed of the patient's cancer diagnosis, and summary discharge letters did not follow any guidelines.

The interventions were complex and, in two studies, involved educating $\mathrm{GPs}^{32,33}$ and/or homecare nurse ${ }^{32}$ about cancer. The unit of randomisation was the patient in both of these studies, although a type of cluster randomisation was used in one. ${ }^{33}$ In one study, once a patient from a practice was randomised, all subsequent patients from the same practice were automatically assigned to the same group. $^{33}$ In the second study patients in the control group could have a GP (or home-care nurse) who had received the educational component of the intervention, or a GP who had not, but this was not taken into account in the analysis. ${ }^{32}$ Two studies included newly diagnosed patients and the intervention was initiated prior to the follow-up 


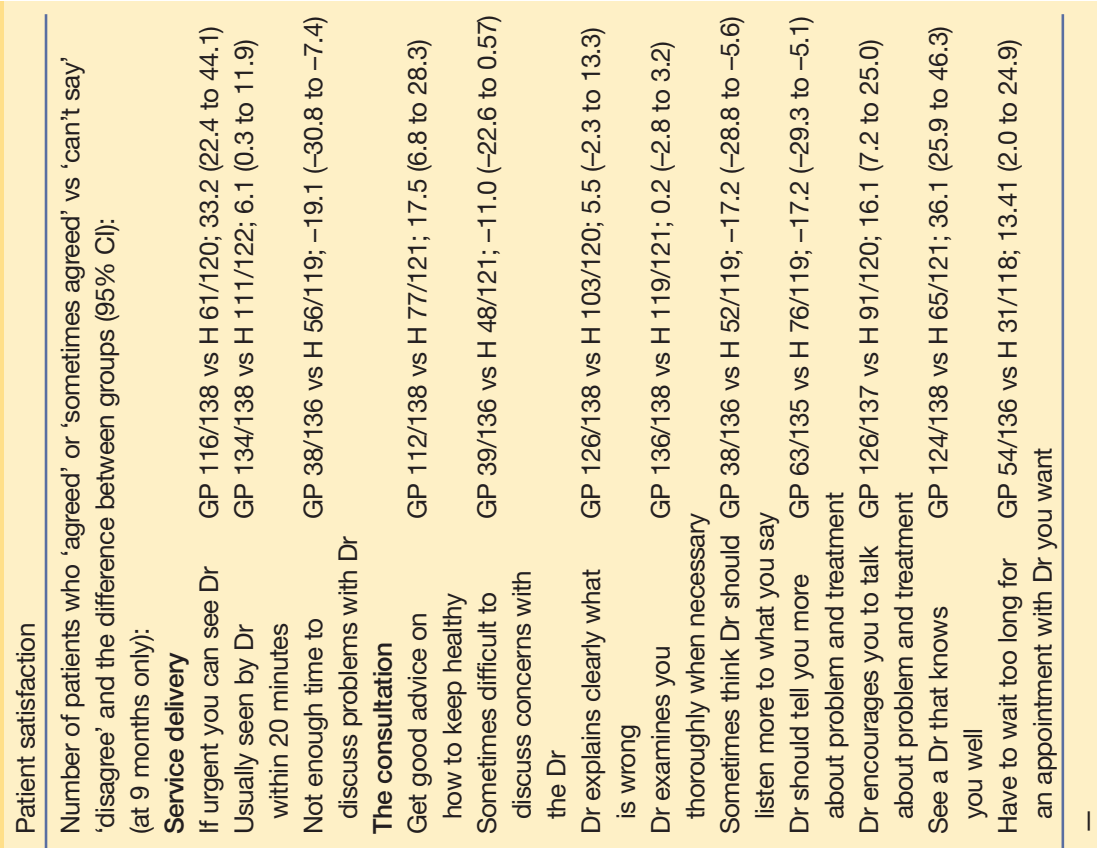

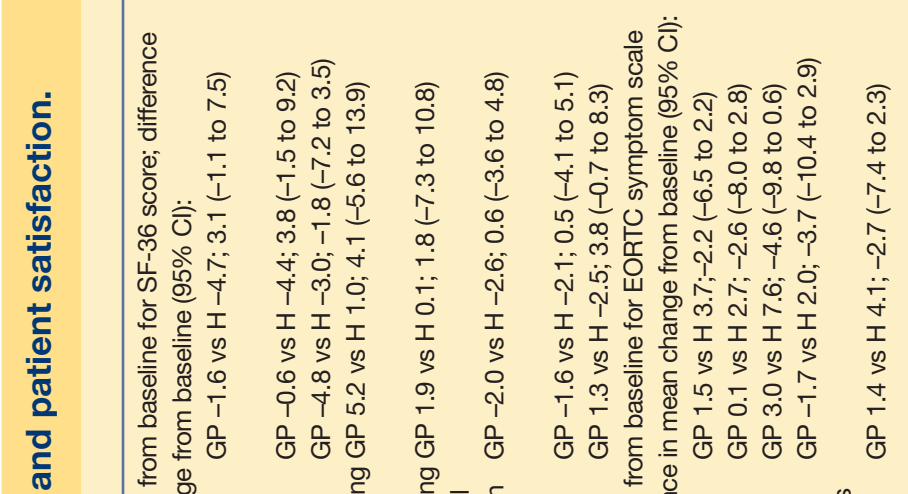

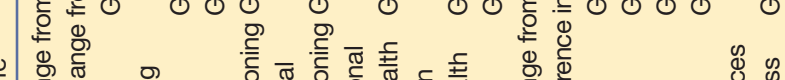

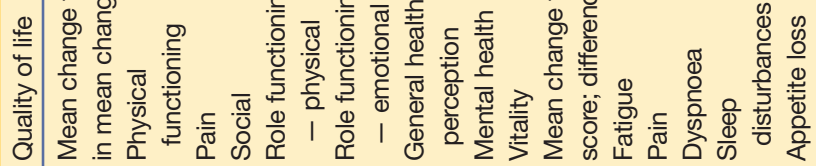

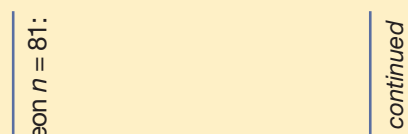

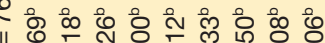

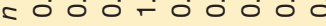

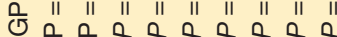
ชิ هI I I I I I I I I

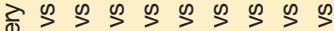

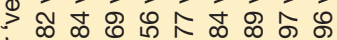

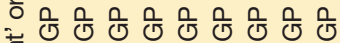

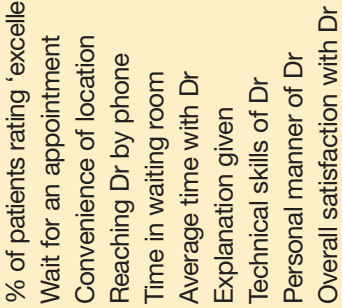

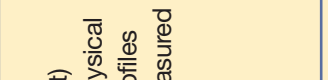

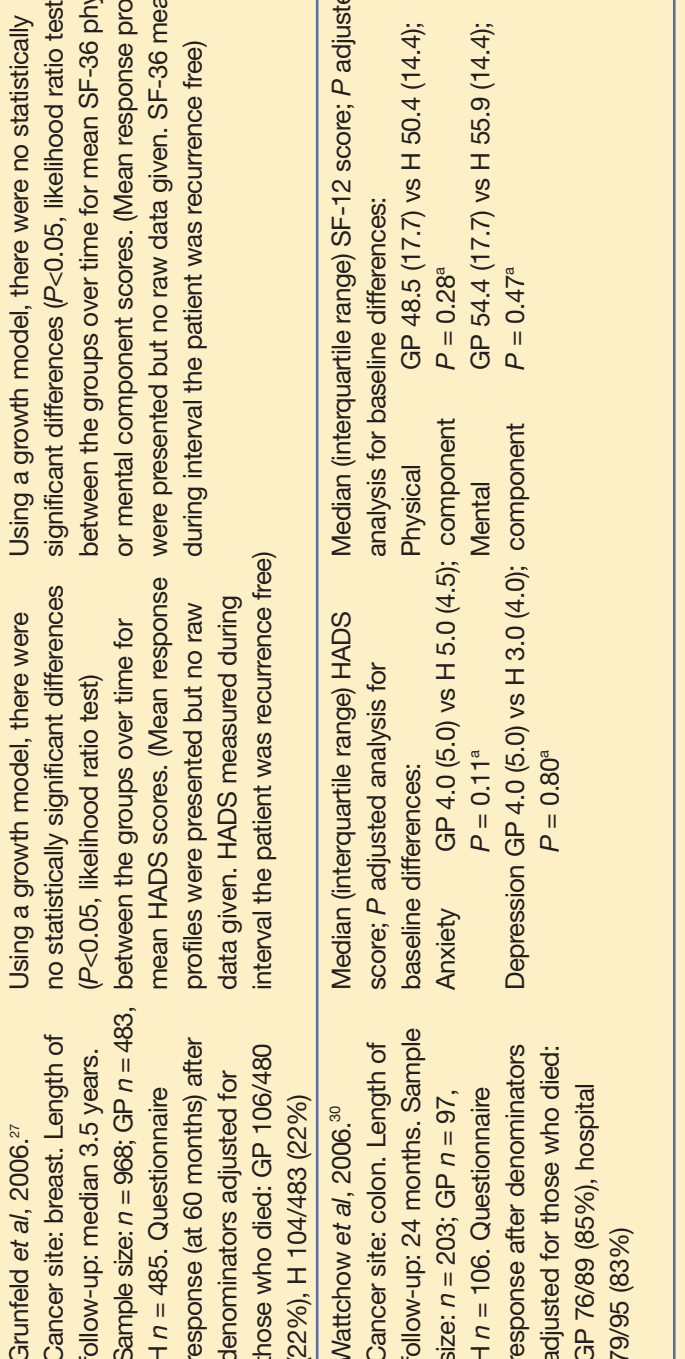




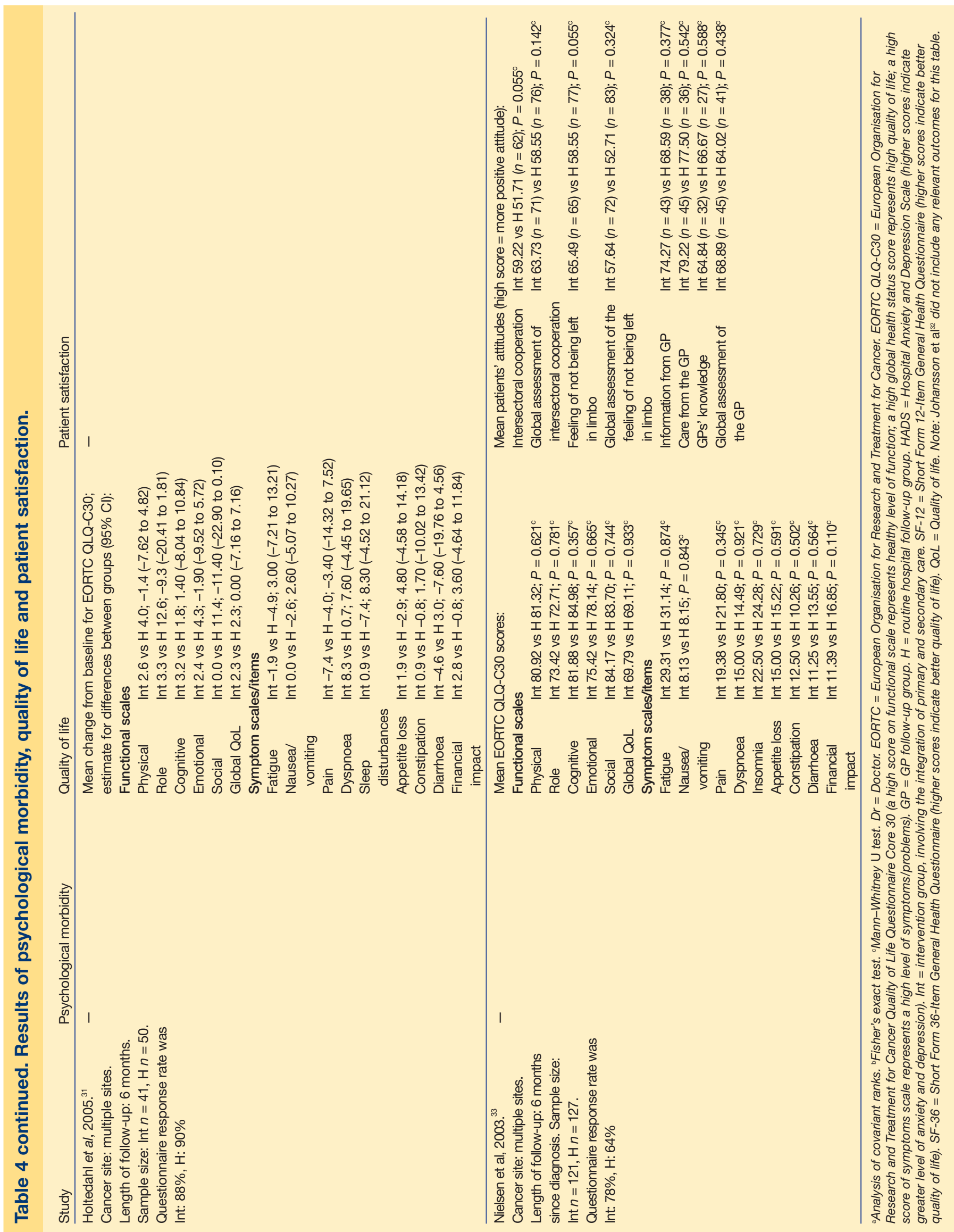




\section{Table 5. Results relating to primary care consultations.}

\section{Study details}

Holtedahl et al, 2005. ${ }^{31}$

Cancer site: multiple sites.

Length of follow-up: 6 months.

Sample size: $\operatorname{lnt} n=41, \mathrm{H} n=50$

\section{Number of patient contacts with primary care} Number of consultations with GP patient reported: Int $13(n=31)$ versus $\mathrm{H} 28$ $(n=46) ;(\mathrm{RR} 0.69,95 \% \mathrm{Cl}=0.43$ to 1.11$)$
Frequency of patient contact with primary care Frequency of GP consultations - reported by GPs: mean number of consultations in the intervention group was 1.68 (ranged 0-8); number of patients not stated. Data for control group not reported. Mean number of consultations - patient reported: Int 1.26 ( $n=31$; range $1-7$ per patient) versus $\mathrm{H} 1.04$ ( $n=46$; range $1-5$ per patient)

Johansson et al, 2001. ${ }^{32}$

Cancer site: multiple site. Length of follow-up: 6 months since diagnosis. Sample size: $\operatorname{lnt} n=260$, $\mathrm{H} n=250$

\section{Nielsen et al, 2003.33}

Cancer site: multiple site. Length of follow-up: 6 months since diagnosis. Sample size: $\operatorname{Int} n=121$, $\mathrm{H} n=127$

Brown et al, 2002.35

Cancer site: breast. Length

of follow-up: 12 months.

Sample size: $n=62$; PI 30, H 31

(one patient did not return questionnaires)

Gulliford et al, 1997. ${ }^{36}$

Cancer site: breast. Length

of follow-up: median 16 months.

Sample size: $n=193$; PI 97, H 96

\section{Kjeldsen et al, 1999. ${ }^{37}$}

Cancer site: colorectal. Length

of follow-up: not stated.

Sample size: $n=320$; PI 161,

H 159. Data based on a subgroup

of 50 patients who were included

in a RCT of frequent versus virtually

no follow-up during $1983-1994^{47}$

Contact with home-care nurse - patient reported: Contact with home-care nurse - patient reported: Int $86(n=203)$ versus $\mathrm{H} 11(n=178) ; P<0.05$, $\chi^{2}$ test (RR $6.86,95 \% \mathrm{Cl}=3.78$ to 12.42 ) frequency of contact with nurse was greater in the intervention group than control (number of patients not stated; $P<0.001$, Mann-Whitney $U$ test)
Total number of GP visits - patient reported (number of visits/patient not reported): PI 53 (4 cancer related) versus $\mathrm{H} 53$ (7 cancer related)

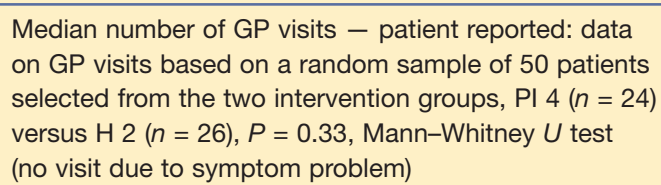
on GP visits based on a random sample of 50 patients selected from the two intervention groups, PI $4(n=24)$ versus $\mathrm{H} 2(n=26), P=0.33$, Mann-Whitney $U$ test (no visit due to symptom problem)

$H=$ routine hospital follow-up group. Int $=$ intervention group. $P I=$ patient-initiated. $R C T=$ randomised controlled trial. $R R=$ relative risk.

period, after completing cancer treatment. ${ }^{32,33}$ The study by Holtendahl et al included patients with primary or relapsing cancer who had completed treatment. ${ }^{31}$ The percentages of eligible patients included in the trials were low and ranged from $41 \%{ }^{31}$ to $47 \%$. $^{33}$

Study quality ranged from poor $^{31}$ to moderate, ${ }^{32,33}$ and all three were poorly reported (Table 2, part b). In the study by Holtedahl et al, patients were randomised to intervention or control using sealed envelopes based on a table of random numbers, but it was not clear when and how envelopes were allocated; therefore, allocation concealment could not be assured. ${ }^{31}$ Although randomisation by Johansson et al was based on a computer-generated allocation schedule and was stratified for diagnosis and stage, allocation concealment was not reported..$^{32}$ More patients with advanced breast cancer were randomised to the intensified primary healthcare group than the control. A type of cluster randomisation was used by Nielsen et al. ${ }^{33}$ Patients in the intervention group were younger ( $n=47,39 \%$, aged 18-49 years) than those in the control group ( $n$ $=32,25 \%$, aged 18-49 years) and more patients in the intervention group ( $n=99,82 \%)$ were treated with curative intent than in the control group ( $n=94,74 \%)$.

Blind data collection and analysis of outcomes were not reported by Johansson et al. ${ }^{32}$ Blinding of outcomes assessment was also not reported by the remaining two studies. The main outcomes were based on data from questionnaires completed by the patient, and it was unclear whether researchers handling data were blinded to treatment allocation. . $^{31,33}$ Sample size ranged from small $(n=91)^{31}$ to moderate $(n=527){ }^{32}$ Thirteen (14\%) patients in the study by Holtedahl et al had relapsing cancer. ${ }^{31}$ The length of follow-up in all three studies was 6 months, although only 3-month data were reported for most outcomes from the study by Johansson et al. ${ }^{32}$

There were no statistically significant differences between the intervention groups in terms of patient wellbeing,,$^{31,33}$ or patient satisfaction relating to GP 
contact, intersectorial cooperation, and patients' feelings of being left in limbo ${ }^{33}$ (Table 4). Two studies found that the intervention was associated with a statistically significant increase in the contact with either the $\mathrm{GP}^{33}$ or home-care nurse ${ }^{32}$ at 6 months (Table 5). However, the Holtedahl et al study found that an intervention comprising two pre-arranged formal consultations with the GP did not result in a significant increase in additional GP visits at 6 months. ${ }^{31}$ There was no statistically significant difference between the intervention groups for hospital admissions and outpatient visits. ${ }^{32}$

\section{Effect of hospital-based patient-initiated or minimal follow-up on primary care}

Three RCTs evaluated the effect of patient-initiated or (virtually) no follow-up on primary care. Two studies included women with breast cancer, and patients in the intervention group were advised to either telephone the nurse, ${ }^{34}$ or request an immediate appointment ${ }^{35}$ if they had any problems. Women received an annual mammogram in both studies. One study included patients who had received treatment for colorectal cancer, and were advised to see their GP if they had any abdominal pain or change in bowel habits lasting more than two weeks. ${ }^{36}$ The quality ranged from poor ${ }^{35}$ to good..$^{34,36}$ There were no important differences between the groups for the number of GP visits ${ }^{35,36}$ or cancerrelated GP referrals. ${ }^{34}$

\section{DISCUSSION}

\section{Summary of main findings}

There were no statistically significant differences between primary and secondary care follow-up of cancer patients (breast or colon) in terms of patient wellbeing, psychological morbidity, and patient satisfaction. However, this may be due to the duration of follow-up and sample size rather than the interventions being equivalent. The findings did not demonstrate any harmful effects of GP-led follow-up. GP-led follow-up for breast cancer was less costly than routine hospital follow-up, due to a difference in physician costs (cost year used 1994). Some interventions that involved improved integration between primary and secondary care resulted in an increase in patient contact with primary care. There were no significant differences between the groups in terms of patient wellbeing and satisfaction. However, these findings are based on poorly reported studies with a short duration of follow-up. The discontinuation of routine hospital follow-up or patient-initiated follow-up did not appear to have an impact on primary care, but this was based on three small RCTs. Overall, the quality of the data was generally poor, and no firm conclusions can be made.

\section{Strengths and limitations of the study}

A strength of the review was the comprehensive literature search. However, to make it manageable, separate searches were carried out of the electronic databases for each cancer site, and a general search was not undertaken using the term 'cancer' (or tumour) alone. Although the searches did identify studies evaluating multiple cancer sites, it is not possible to be certain that none were missed. However, database searches were supplemented by a search of conference proceedings and reference lists of included studies and other reviews, which were not narrowed by cancer site.

The review concentrated on the use of primary care as an alternative setting for cancer follow-up and did not address any other issues relating to follow-up.

Because of the small number of relevant studies, it was not possible to assess for any publication bias. The inconsistent methods used to analyse and report most outcome measures meant that no data could be pooled in a meta-analysis.

There are limitations of the available evidence relating to primary-versus secondary-care follow-up, as it is small and only covers two cancer sites: the breast ${ }^{3,27}$ and colon. ${ }^{30}$ Limitations of the included noninferiority RCT meant that the evidence for assessing the equivalence of the primary and secondary care settings for breast cancer follow-up was lacking. There is also a paucity of economic evidence on primary versus secondary care follow-up. Further evidence relating to the effectiveness and costeffectiveness of primary care follow-up may be provided by an RCT of primary care follow-up for cutaneous melanoma, ${ }^{29}$ and an ongoing RCT of symptomatic follow-up of colorectal cancer in primary care augmented with monitoring of tumour markers or intensive imaging in hospital (http://www.facs.soton.ac.uk/).

The length of follow-up and sample size were insufficient to measure delayed diagnosis of recurrences or survival rates, which means that the impact of various types of follow-up on such outcomes is not clear. Duration of follow-up is also likely to affect the outcome of patient satisfaction, as patients are likely to have a different perspective of their follow-up needs during the first 2 years after completing treatment, than later in their cancer journey. ${ }^{37}$

The data-collection tools used for psychological morbidity, health-related quality of life, and patient satisfaction were limited. Although the Hospital Anxiety and Depression Scale and Short Form-36 are good instruments to measure global function, they are not designed to measure cancer survivor symptoms. On the whole, patient satisfaction 
questionnaires were not well developed, and the response rates were poor, making the findings potentially unreliable.

The patient population evaluated in the comparative studies may not have been representative of the population attending cancer follow-up as a whole, because patients who did not want primary care follow-up may not have been randomised.

\section{Comparison with existing literature}

Other reviews of cancer follow-up have included comparisons between different models of follow-up, providers, and location. Only one other systematic review looking at primary versus secondary care follow-up has been identified..$^{38}$ This review evaluated RCTs of alternative methods of follow-up in breast cancer, including reduced frequency of visits. It included seven RCTs: two that compared primary with secondary care follow-up, ${ }^{3,27}$ two that evaluated nurse-led patient-initiated follow-up, ${ }^{34,39}$ one that evaluated nurse-led routine follow-up, ${ }^{40}$ and two that examined different frequencies of follow-up..$^{35,41}$ The authors concluded that all trials were of inadequate power or duration to establish the ideal frequency of appointments or safety of alternative models of follow-up, but the alternative methods of follow-up had no detrimental effect on satisfaction or outcome. Two further reviews evaluating the effectiveness of breast cancer follow-up found that patient survival and quality of life were not affected by location of care. ${ }^{12,14}$ A systematic review of follow-up for cutaneous melanoma found no studies that examined differences between different providers or locations of follow-up. ${ }^{16}$

Previous systematic reviews found that the only effective follow-up procedures in breast cancer were mammography and physical examination. ${ }^{12,13,42}$ Systematic reviews of colorectal cancer found that intensive hospital follow-up led to an overall survival benefit of about $20 \%$ when compared with lessintensive follow-up. ${ }^{43,44}$ The survival benefit appeared to be associated with the measurement of carcinoembryonic antigen combined with liver imaging. A systematic review of follow-up for cutaneous melanoma found no evidence to support high-intensity follow-up. Only medical history and physical examination appeared to be cost-effective. ${ }^{16}$ However, new technological developments in followup methods may change the picture of what to do.

\section{Implications for future research and clinical practice}

With further training for GPs, rapid access to hospital specialists, and annual mammography, breast cancer follow-up would be feasible in primary care.
However, further psychosocial studies are needed to determine its acceptability. The follow-up of of colon cancer might also be feasible, but the evidence base is limited. Results of research in progress are awaited (http://www.facs.soton.ac.uk/). Primary-care followup for breast cancer might also be cost-effective. However, it is dependent on the unit cost of GP care, and would require additional funding as it is not a core activity. The willingness of primary care to undertake this additional work is unknown, but interventions that improved communication between primary and secondary care were found to increase GP involvement in cancer care. ${ }^{31-33}$ Poor communication between primary and secondary care was seen as a barrier in the current authors' joint publication of qualitative studies, ${ }^{22}$ and improved communication is a key recommendation of the National Institute for Health and Clinical Excellence guidelines on breast and colorectal cancer. ${ }^{45,46}$ The willingness of GPs to undertake this role may also be hampered by a perceived lack of specialist knowledge. ${ }^{22}$ Only one study of primary care follow-up (breast cancer) incorporated a pretrial education session of cancer follow-up for GPs. ${ }^{3}$ Continued professional education in oncology will be needed if this role is to be extended.

Further RCTs are needed of primary versus secondary care follow-up in cancer where the ideal hospital-based follow-up is transferable to a primary care setting. The studies need to be of sufficient size and duration to ensure that important differences between the intervention groups are identified. They should also include robust psychosocial outcome measures. Future research should include a healtheconomic analysis that takes into account the current cost of general practice and the additional funding required for this non-core activity.

\section{Funding body}

Cancer Research UK (Ref C8350/A4543). The funding source had no involvement in the study

\section{Ethical approval}

Not applicable

\section{Competing interests}

The authors have stated that there are none

\section{Acknowledgments}

We would like to thank Stephen Duffy for his advice on searching databases and developing the search strategy, Bernadette Coles and Hilary Kitcher for checking some of the original search strategies, Madeline Pasterfield and Jennifer Wilkinson for technical support including carrying out the update searches, and Alison Eastwood for peer reviewing the protocol and draft final report. We would also like to thank the following people for sending us further information about the studies they had conducted: Kinta Beaver, Gillian Knowles, Karen Cox, Christine Mcllvenney, and Stephen Barclay. Thanks also to Richard Bailey and his staff at the John Spalding Library for their help in retrieving manuscripts.

\section{Discuss this article}

Contribute and read comments about this article on the Discussion Forum: http://www.rcgp.org.uk/bjgp-discuss 


\section{REFERENCES}

1. Donnelly P, Hiller L, Bathers S, et al. Questioning specialists' attitudes to breast cancer follow-up in primary care. Ann Oncol 2007; 18(9): 1467-1476.

2. Pascoe SW, Neal RD, Allgar VL, et al. Psychosocial care for cancer patients in primary care - recognition of opportunities for cancer care. Fam Pract 2004; 21(4): 437-442.

3. Grunfeld E, Mant D, Yudkin P, et al. Routine follow up of breast cancer in primary care: randomised trial. BMJ 1996; 313(7058): 665-669.

4. Campbell N, MacLeod U, Weller D. Primary care oncology: essential if high quality cancer care is to be achieved by all. Fam Pract 2002; 19(6): $577-578$

5. Koinberg IL, Holmberg L, Fridlund B. Breast cancer patients' satisfaction with a spontaneous system of check-up visits to a specialist nurse. Scand J Caring Sci 2002; 16(3): 209-215.

6. Rozmovits L, Rose P, Ziebland S. In the absence of evidence, who chooses? A qualitative study of patients' needs after treatment for colorectal cancer. J Health Serv Res Policy 2004; 9(3): 159-164.

7. Bradburn J, Maher J, Adewuyidalton R, et al. Developing clinical-trial protocols - the use of patient focus groups. Psychooncology 1995; 4: 107-112.

8. Cox K, Wilson E, Heath W, et al. Preferences for follow-up after treatment for lung cancer. Cancer Nurs 2006; 29(3): 176-187.

9. Beaver K, Luker K. Follow-up in breast cancer clinics: reassuring for patients rather than detecting recurrence. Psychooncology 2005; 14(2): 94-101.

10. Pennery E, Mallet J. A preliminary study of patients' perceptions of routine follow-up after treatment for breast cancer. Eur J Oncol Nurs 2000; 4(3): 138-145.

11. Allen A. The meaning of the breast cancer follow-up experience for the women who attend. Eur J Oncol Nurs 2002; 6(3): 155-161.

12. Rojas MP, Telaro E, Russo A, et al. Follow-up strategies for women treated for early breast cancer. Cochrane Database Syst Rev 2000; (4): CD001768.

13. Somerfield MR, Schapira DV, Davidson NE, et al. Recommended breast cancer surveillance guidelines. J Clin Oncol 1997; 15: 2149-2156.

14. Collins RF, Bekker HL, Dodwell DJ. Follow-up care of patients treated for breast cancer: a structured review. Cancer Treat Rev 2004; 30(1): $19-35$.

15. Botteman MF, Pashos CL, Redaelli A, et al. The health economics of bladder cancer: a comprehensive review of the published literature. Pharmacoeconomics 2003; 21(18): 1315-1330.

16. Francken AB, Bastiaannet E, Hoekstra HJ. Follow-up in patients with localised primary cutaneous melanoma. Lancet Oncol 2005; 6(8): 608-621.

17. Kerr D, Bevan H, Gowland B, et al. Redesigning cancer care. $B M$ J 2002; 324(7330): 164-166.

18. National Institute for Health and Clinical Excellence. Improving outcomes for people with skin tumours including melanoma - the manual. London: NICE, 2006.

19. National Institute for Health and Clinical Excellence. Prostate cancer: diagnosis and treatment. London: NICE, 2008.

http://www.nice.org.uk/guidance/index.jsp?action=download\&o=396 87 (accessed 17 Feb 2009).

20. Neal RD. NICE prostate cancer clinical guideline: implications for primary care. Br J Gen Pract 2008; 58(554): 607-608

21. Weller D, McGorm K. GP vs hospital follow-up for cancer patients. Aust Fam Physician 1999; 28: 356-359.

22. Lewis RA, Neal RD, Hendry M, et al. Patients' and healthcare professionals' views of cancer follow-up: systematic review. $\mathrm{Br} \mathrm{J} \mathrm{Gen}$ Pract 2009; 59(559): 533-540.

23. Lewis R, Neal RD, Williams NH, et al. Nurse-led vs conventional physician-led follow-up for patients with cancer: systematic review. J Adv Nurs 2009; 65: 706-723.

24. Downs SH, Black N. The feasibility of creating a checklist for the assessment of the methodological quality both of randomised and non-randomised studies of health care interventions. J Epidemiol Community Health 1998; 52(6): 377-384.

25. Deeks JJ, Dinnes J, D’Amico R, et al. Evaluating non-randomised intervention studies. Health Technol Assess 2003; 7(27): iii-x, 1-173.

26. Drummond MF, Jefferson TO. Guidelines for authors and peer reviewers of economic submissions to the BMJ. BMJ 1996; 313(7052): 275-283.

27. Grunfeld E, Levine MN, Julian JA, et al. Randomised trial of longterm follow-up for early-stage breast cancer: a comparison of family physician versus specialist care. J Clin Oncol 2006; 24(6): 848-854.

28. Loberiza FR, Villaneuva MH, et al. Effect of follow-up provider source on outcomes of patients with haematologic malignancies receiving haematopoietic stem cell transplantation [abstract]. J Clin Oncol 2006 24 (Suppl 18S): 6112.

29. Murchie P, Hannaford PC, Nicolson MC, et al. Attending the general practitioner for routine melanoma follow-up: the practical experiences of participants in an RCT. In: The Society for Academic Primary Care 35th Annual Scientific Meeting; 2006 12-14 July 2006, Keele University, UK.

30. Wattchow DA, Weller DP, Esterman A, et al. General practice vs surgical-based follow-up for patients with colon cancer: randomised controlled trial. Br J Cancer 2006; 94(8): 1116-1121.

31. Holtedahl K, Norum J, Anvik T, Richardsen E. Do cancer patients benefit from short-term contact with a general practitioner following cancer treatment? A randomised controlled study. Support Care Cancer 2005; 13(11): 949-956.

32. Johansson B, Holmberg L, Berglund G, et al. Reduced utilisation of specialist care among elderly cancer patients: a randomised study of a primary healthcare intervention. Eur J Cancer 2001; 37(17): 2161-2168.

33. Nielsen JD, Palshof T, Mainz J, Jensen AB, Olesen F. Randomised controlled trial of a shared care programme for newly referred cancer patients: bridging the gap between general practice and hospital. Qual Saf Health Care 2003; 12(4): 263-272.

34. Brown L, Payne S, Royle G. Patient initiated follow up of breast cancer. Psychooncology 2002; 11(4): 346-x5.

35. Gulliford T, Opomu M, Wilson E, et al. Popularity of less frequen follow up for breast cancer in randomised study: initial findings from the hotline study. BMJ 1997; 314(7075): 174-177.

36. Kjeldsen BJ, Thorsen H, Whalley D, Kronborg O. Influence of followup on health-related quality of life after radical surgery for colorectal cancer. Scand J Gastroenterol 1999; 34(5): 509-515.

37. Adewuyi-Dalton R, Ziebland S, Grunfeld E, Hall A. Patients' views of routine hospital follow-up: a qualitative study of women with breast cancer in remission. Psychooncology 1998; 7(5): 436-439.

38. Montgomery DA, Krupa K, Cooke TG. Alternative methods of follow up in breast cancer: a systematic review of the literature. $\mathrm{Br} J$ Cancer 2007; 96(11): 1625-1632.

39. Koinberg IL, Fridlund B, Engholm GB, Holmberg L. Nurse-led follow-up on demand or by a physician after breast cancer surgery: a randomised study. Eur J Oncol Nurs 2004; 8(2): 109-117.

40. Baildam A, Keeling F, Noblet M, et al. Nurse led follow up clinics for women treated for primary breast cancer: a randomised controlled trial. Eur J Cancer 2002; 38: S136.

41. Kokko R, Hakama M, Holli K. Follow-up cost of breast cancer patients with localized disease after primary treatment: a randomized trial. Breast Cancer Res Treat 2005; 93(3): 255-260.

42. Edelman MJ, Meyers FJ, Siegel D. The utility of follow-up testing after curative cancer therapy: a critical review and economic analysis. J Gen Intern Med 1997; 12(5): 318-331.

43. Jeffery GM, Hickey BE, Hider P. Follow-up strategies for patients treated for non-metastatic colorectal cancer. Cochrane Database Syst Rev 2007; (1): CD002200.

44. Renehan AG, Egger M, Saunders MP, O'Dwyer ST. Impact on survival of intensive follow up after curative resection for colorectal cancer: systematic review and meta-analysis of randomised trials. BMJ 2002; 324(7341): 813-816.

45. National Institute for Clinical Excellence. Improving outcomes in colorectal cancers - the manual update. London: National Institute of Clinical Excellence, 2004.

46. National Institute for Clinical Excellence. Improving outcomes in breast cancer - the manual update. London: National Institute for Clinical Excellence, 2002.

47. Kjeldsen BJ, Kronborg O, Fenger C, Jorgensen OD. A prospective randomized study of follow-up after radical surgery for colorectal cancer. Br J Surg 1997; 84(5): 666-669. 


\section{Appendix 1. Search strategy.}

Search strategy

The following search strategy for breast cancer was used in MEDLINE (using the OVID interface), and subsequently translated and adapted for use in other databases (and for other cancer sites).

1. Patient discharge/

2. ((followup\$ or follow-up\$ or follow up or follow ups) adj4 (discharg\$ or discontin\$)).ti,ab.

3. ((checkup\$ or check-up\$ or check up\$) adj4 (discharg\$ or discontin\$)).ti,ab.

4. ((followup\$ or follow-up\$ or follow up or follow ups) adj4 (stop or stops or stopping or stopped)).ti,ab.

5. ((followup\$ or follow-up\$ or follow up or follow ups) adj4 (cease or ceases or ceasing or ceased or cessation)).ti,ab.

6. ((checkup\$ or check-up\$ or check up or check ups) adj4 (cease or ceases or ceasing or ceased or cessation)).ti,ab.

7. ((followup\$ or follow-up\$ or follow up or follow ups) adj4 (end or ends or ending or ended)).ti,ab.

8. ((checkup\$ or check-up\$ or check up or check ups) adj4 (end or ends or ending or ended)).ti,ab.

9. ((followup\$ or follow-up\$ or follow up or follow ups) adj4 terminat\$).ti,ab.

10.(followup\$ or follow-up\$ or follow up or follow ups) adj4 finish\$).ti,ab.

11.(followup\$ or follow-up\$ or follow up or follow ups) adj4 withdraw\$).ti,ab.

12.((followup\$ or follow-up\$ or follow up or follow ups) adj4 (cut-off or cut off)).ti,ab.

13.continuity of patient care/

14.((minimal or conventional) adj2 (surveillance $\$$ or followup\$ or follow-up\$ or follow up or follow ups)).ti,ab.

15.(routine adj2 (followup\$ or follow-up\$ or follow up or follow ups or visit\$)).ti,ab.

16.(routine adj2 (checkup\$ or check-up\$ or check up or check ups)).ti,ab.

17.(surveillance $\$$ adj2 (recur $\$$ or relaps $\$$ or protocol $\$$ or routine $\$$ or regular $\$$ or followup $\$$ or follow-up $\$$ or follow up or follow ups)).ti,ab.

18.(surveillance\$ adj2 (test or tests or testing or tested or hospital or outpatient\$ or out-patient or out patient\$ or standard\$)).ti,ab.

19.((intensive or frequent or aggressive) adj2 (surveillance\$ or followup\$ or follow-up\$ or follow up or follow ups)).ti,ab.

20.active\$ monitor\$.ti,ab.

21.(routine adj2 exam\$).ti,ab.

22.(routine adj2 review\$).ti,ab.

23.(routine\$ adj2 (test or tests or testing or tested)).ti,ab.

24.(outpatient\$ adj2 (followup\$ or follow-up\$ or follow up or follow ups)).ti,ab.

25.(outpatient\$ adj2 (checkup\$ or check-up\$ or check up or check ups)).ti,ab.

26.(systematic adj2 (followup\$ or follow-up\$ or follow up or follow ups)).ti,ab.

27.(scheduled adj2 (followup\$ or follow-up\$ or follow up or follow ups)).ti,ab.

28.(scheduled adj2 (checkup\$ or check-up\$ or check up or check ups)).ti,ab.

29.(regular\$ adj2 (followup\$ or follow-up\$ or follow up or follow ups)).ti,ab.

30.(regular\$ adj2 (checkup\$ or check-up\$ or check up or check ups)).ti,ab.

31.(specialist\$ adj2 (followup\$ or follow-up\$ or follow up or follow ups)).ti,ab.

32.(hospital\$ adj2 (followup\$ or follow-up\$ or follow up or follow ups)).ti,ab.

33.(hospital\$ adj2 (checkup\$ or check-up\$ or check up or check ups)).ti,ab.

34.((clinic or clinics) adj2 (followup\$ or follow-up\$ or follow up or follow ups)).ti,ab. 


\section{Appendix 1 continued. Search strategy.}

35.((clinic or clinics) adj2 (checkup\$ or check-up\$ or check up or check ups)).ti,ab.

36.(initiated adj2 (followup\$ or follow-up\$ or follow up or follow ups)).ti,ab.

37.(initiated adj2 (checkup\$ or check-up\$ or check up or check ups)).ti,ab.

38.((general practitioner\$ or GP\$ or practice or physician) adj2 (followup\$ or follow-up\$ or follow up or follow ups)).ti,ab.

39.((general practitioner\$ or GP\$ or practice or physician) adj2 (checkup\$ or check-up\$ or check up or check ups)).ti,ab.

40.((telephone or phone\$) adj2 (followup\$ or follow-up\$ or follow up or follow ups)).ti,ab.

41.((telephone or phone\$) adj2 (checkup\$ or check-up\$ or check up or check ups)).ti,ab.

42.((followup\$ or follow-up\$ or follow up or follow ups) adj2 (secondary or primary)).ti,ab.

43.((checkup\$ or check-up\$ or check up or check ups) adj2 (secondary or primary)).ti,ab.

44.(nurse $\$$ adj2 (followup\$ or follow-up\$ or follow up or follow ups)).ti,ab.

45.(nurse $\$$ adj2 (checkup\$ or check-up\$ or check up or check ups)).ti,ab.

46.(standard\$ adj2 (followup\$ or follow-up\$ or follow up or follow ups)).ti,ab.

47.(standard\$ adj2 (checkup\$ or check-up\$ or check up or check ups)).ti,ab.

48.(followup\$ or follow-up\$ or follow up or follow ups) adj2 regime\$).ti,ab.

49.((followup\$ or follow-up\$ or follow up or follow ups) adj2 (postsurgery or post-surgery or post surgery or postsurgical\$ or post-surgical\$ or post surgical\$ or postoperat\$ or post-operat $\$$ or post operat\$)).ti,ab.

50.((checkup\$ or check-up\$ or check up or check ups) adj2 (postsurgery or post-surgery or post surgery or postsurgical $\$$ or post-surgical\$ or post surgical\$ or postoperat $\$$ or post-operat $\$$ or post operat $\$)) . t i, a b$.

51.(followup\$ or follow-up\$ or follow up or follow ups) adj2 appointment\$).ti,ab.

52.((checkup\$ or check-up\$ or check up or check ups) adj2 appointment\$).ti,ab.

53.or/1-52

54.exp Breast Neoplasms/

55.((breast or breasts or mammar $\$$ ) adj3 (cancer $\$$ or neoplas $\$$ or malignan $\$$ or carcinoma $\$$ or sarcoma $\$$ or oncolog $\$$ or tumo? $\$$ or adenocarcinoma or infiltrat\$ or medullary or intraductal)).ti,ab.

56.((breast or breasts or mammar\$) adj3 (duct or ducts or ductal)).ti,ab.

57.((breast or breasts or mammar\$) adj3 (lobule\$ or lobe or lobes or lobular)).ti,ab.

58.((breast or breasts or mammar\$) adj3 (metastas\$ or metastatic\$)).ti,ab.

59.54 or 55 or 56 or 57 or 58

60.53 and 59

The strategy is based on the one used for an unpublished scoping review (looking at follow-up for breast cancer) undertaken by the Centre for Reviews and Dissemination, University of York as part of a project with the National Cancer Research Network

Coordinating Centre. 